\title{
La modélisation conceptuelle comme aide à la construction de villes résilientes
}

\section{Conceptual modelling as guidance for helping to build resilient cities}

\author{
Laurie Boschetti ${ }^{1}$, Damienne Provitolo ${ }^{1}$, Emmanuel Tric ${ }^{1}$ \\ ${ }^{1}$ Université Côte d'Azur, CNRS, OCA, IRD, UMR Géoazur, Laurie.boschetti@geoazur.unice.fr, \\ damienne.provitolo@geoazur.unice.fr, emmanuel.tric@geoazur.unice.fr
}

RÉSUMÉ. La résilience est un concept mobilisé par de nombreuses disciplines, et qui est depuis une dizaine d'année mobilisé par les cindyniques. II est encore difficile aujourd'hui de trouver un accord sur sa définition et sur sa mise en application au sein de la communauté scientifique. Nous ne nous attardons pas ici sur les différentes définitions existantes, mais nous nous intéressons plus spécifiquement aux différents modèles de mise en application de la résilience que l'on retrouve dans la littérature. Nous nous focalisons plus particulièrement sur les modèles systémiques de résilience, qu'ils soient génériques, ou opérationnels et applicables par les acteurs. Les travaux retenus (Panarchy [GUN 02], Cycle Adaptatif [WAL 04], Resilience Alliance [RES 10], Spatial Decision Support System [BAL 13]), ne répondent que partiellement à notre objectif, qui est d'obtenir un cadre opérationnel d'aide à la construction des villes résilientes. Nous avons donc cherché à construire une nouvelle méthodologie permettant de construire des territoires et des sociétés résilientes. Nous proposons ainsi un nouveau modèle d'analyse de la résilience qui se base sur les travaux de nos prédécesseurs : le modèle CREAA. Celui-ci se focalise sur deux temporalités : l'avant et l'après événement, et est composé de quatre modules s'articulant autour de cibles majeurs déterminées en amont de l'étude, et permettant d'adapter le modèle en fonction de différents cas d'études (différents territoires et différents aléas).

ABSTRACT. The resilience concept is use by many fields of study, and has been more and more use by the risk science in the last ten years. It is still difficult today to give a single definition and to found an agreement on its meaning and its application inside the scientific community. In this paper, we are not explaining the different definitions but we are taking an interest in models of implementation of resilience that are available in the literature. We are more specifically focusing on systemic patterns, which are generic or operational and applicable by stakeholders. Research work that we had selected (Panarchy [GUN 02], Cycle Adaptatif [WAL 04], Resilience Alliance [RES 10], Spatial Decision Support System [BAL 13] answered partially to our aim, which is to found an operational framework that helps to build resilient cities. We looked for a new methodology that could be used to build territories and societies resilience. Therefore, we are suggesting a new resilience model, relying on previously mentioned studies: the CREAA model. This one is focusing on two temporalities: before and after the event. It is made up of four units, that has been built around key targets, specific to every studies, and that allowing the model to adapt different situations (different territories and different hazards).

MOTS-CLÉS. Résilience, modèle de résilience, construction de la résilience, modèle CREAA, Panarchy, Cycle adapatatif, Resilience Alliance, DS3.

KEYWORDS. Resilience, resilience model, building resilience, CREAA model, Panarchy, Adaptive cycle, Resilience Alliance, DS3.

\section{Introduction}

Ces quarante dernières années ont été marquées par un accroissement important du nombre de catastrophes, et ce particulièrement dans les villes qui hébergent plus de la moitié de la population mondiale. Ces tendances ne devraient pas s'inverser dans les années à venir, car les facteurs de risque restent nombreux : changements climatiques, tensions géopolitiques, risques liés aux avancées de la technologie et aux besoins des sociétés, croissance démographique et pauvreté, dégradation de l'environnement et pression urbaine etc. [URD 11].

Pour faire face à ces risques multiples, la résilience ${ }^{1}$ est devenue lors du « Cadre d'Action de Hyogo pour 2005-2015 : pour des nations et des collectivités résilientes face aux catastrophes », le nouveau

\footnotetext{
${ }^{1}$ « Resilience is the ability to prepare and plan for, absorb, recover from, and more successfully adapt to adverse events. Enhanced resilience allows better anticipation of disasters and better planning to reduce disaster losses -rather than waiting for an event to occur and paying for it afterward » National Academy [NAT 12]. 
cadre, le nouveau référentiel de la politique de gestion des catastrophes. Il s'agit là du principal instrument que les États membres des Nations unies ont adopté pour réduire les risques de catastrophe.

Toutefois, force est de constater que ce concept reste, douze ans après la conférence mondiale de Hyogo, encore peu opérationnel pour construire les résiliences des territoires et des sociétés. Le plus souvent les études sont empiriques, réalisées à différentes échelles et portent sur des systèmes aussi distincts qu'un pays, une ville, un quartier, une communauté humaine ou une entreprise. En l'absence de cadre méthodologique commun, les comparaisons pour identifier les villes résilientes sont donc difficiles. De plus, dans la majorité des recherches, la difficulté de mettre en œuvre une démarche de résilience opérationnelle apparaît clairement [O'RO 07]. Quelques rares tentatives ont mobilisé les modèles stylisés du Cycle adaptatif [WAL 04], de Panarchy [GUN 02] et la méthode proposée par le groupe de recherche Resilience Alliance [RES 10]. Comme nous le montrerons dans la $1^{\text {ere }}$ partie de cet article, dans la réalité, ces modèles restent encore peu mobilisés dans le champ des risques et des catastrophes.

L'un des défis actuels dans le domaine de la sécurité ${ }^{2}$ des territoires et des populations, défi que nous souhaitons aborder dans le cadre de cet article, consiste donc à proposer des cadres méthodologiques à portée opérationnelle pour soutenir les acteurs opérationnels et institutionnels dans leur démarche de construction de territoires résilients. Construire la résilience territoriale signifie que cette dernière n'est pas une qualité mais un processus. En étant appréhendé comme un processus, les réponses résilientes ne se limitent plus uniquement à l'urgence de l'intervention, à la reconstruction après un choc. Elles visent également à anticiper les menaces potentielles et leurs répercussions, à permettre aux organisations (notamment celles constituées d'acteurs opérationnels et institutionnels) et aux populations de se préparer aux risques de catastrophe, à se protéger contre les catastrophes, mais aussi à faire face à l'urgence d'une situation afin de parer à d'éventuelles crises. La construction de territoires et populations résilients amène ainsi à discerner des actions et des pratiques liées à différentes échelles de temps [MAZ 17], celles s'inscrivant dans la temporalité de la prévention des risques de catastrophes, celle de la gestion des catastrophes et de l'urgence, celles de la remise en état, de la reconstruction, voire de la réorganisation des territoires, celles de la consolidation des pratiques résilientes, celles des retours d'expérience afin de tirer les enseignements d'événements passés dans une vision prospective permettant de réintroduire "dans la notion même de risque le processus d'apprentissage (voire d'innovation) que peut constituer la situation postcatastrophe" [GEN 16].

Deux événements sont le point de départ de notre réflexion sur la mise en action de la résilience des villes et des populations aux prises avec des événements soudains, à dynamique rapide et se produisant "par surprise".

Le 3 octobre 2015, des précipitations soudaines et intenses (on relève par exemple 106mm en une heure à la station Météo France de Cannes/Mandelieu ou 74mm en une heure à Nice, ces deux valeurs constituent les records des stations pour les cumuls horaires depuis le début des relevés) ont engendré une catastrophe urbaine sur le littoral de la Côte d'Azur et plus spécifiquement entre les communes de Mandelieu et Nice. Ces pluies violentes ont provoqué le débordement de plusieurs cours d'eau et de nombreux ruissellements urbains. Cet événement met en difficulté un territoire s'étendant sur plus de quarante km, de Théoule-sur-Mer à Nice, et prend très rapidement une tournure catastrophique. Cette tournure se lit notamment dans la désorganisation du territoire, les ruptures de communication terrestre, la saturation de la centrale téléphonique du SDIS 06, la difficulté pour les pompiers et les responsables locaux d'assurer leurs missions de façon habituelle, notamment celles de prise en charge des populations, ou encore dans la médiatisation de l'événement. Vingt personnes ont perdu la vie, et ce du fait essentiellement de comportements inadaptés à la situation de montée des eaux (descente des personnes dans des points bas comme des sous-sol ou des tunnels). Au niveau du bilan économique, dix-sept mille véhicules ont été endommagés ou détruits, les dégâts s'élèvent à six cent quatre-vingt-dix millions d'euros (quatre-vingt-cinq millions d'euros pour des collectivités locales sur les équipements

\footnotetext{
${ }^{2}$ L'approche sécuritaire, questionnée sous l'angle de la résilience [LAG 09], dépasse désormais largement les seules idées de protection et de contrôle. 
publics et six cent cinq millions d'euros de dégâts assurés d'après l'Association Française de l'Assurance), et trente-deux communes des départements des Alpes-Maritimes et du Var ont été placées en état de Catastrophes Naturelles dès le 7 octobre 2015.

Une partie de ce même territoire a été frappé quelques mois plus tard, lors de la fête nationale du 14 juillet 2016, par un aléa de tout autre nature, un attentat terroriste. L'effet de surprise est l'élément marquant d'un attentat, d'autant plus qu'il frappe la plupart du temps des civils dans des lieux de la vie courante à forte densité humaine, comme les stations de métro, les gares, les aéroports ou les lieux de festivités. Ce fut le cas à Nice, où la scène de l'attaque s'étend sur près de deux km sur la Promenade des Anglais. Le bilan humain est très lourd avec quatre-vingt-six personnes ayant perdu la vie et des centaines de blessés.

Inondation et attentat, deux événements ayant a priori peu de points communs si l'on s'attarde uniquement sur l'origine de la catastrophe : l'une est déclenchée par une action naturelle, l'autre par une action humaine. Mais ces événements présentent également des caractéristiques similaires : événements difficilement prévisibles et localisables précisément, événements dont l'emprise spatiale est réduite comparativement à des catastrophes obéissant à un modèle zonal (ex. une pandémie), brutaux dans leur déclenchement, à cinétique rapide. Evénements auxquels les victimes sont peu préparées, sont parfois difficilement accessibles (isolées, voire immergées dans leurs habitations) et dispersées sur le territoire du fait de l'emprise spatiale de l'inondation, ou des mouvements de fuite qui peuvent être observés lors des attentats. Le caractère inattendu de ces deux catastrophes, auquel s'ajoute le caractère inhabituel d'action terroriste sur la métropole azuréenne, amène à questionner les actions et pratiques de résiliences aux échelles individuelles, collectives, sociétales et interorganisationnelles. Ces pratiques ont trait à des domaines variés, notamment ceux de l'information (disponible, transmise, comprise) lors de catastrophes, ceux de l'adoption de comportements adaptés à la situation, ceux de la protection, qu'elle soit structurelle (adaptation de l'architecture, ouvrages de défense) ou non structurelle (comment se protéger individuellement), ceux de la gouvernance entre les acteurs institutionnels et opérationnels.

Ce constat d'ensemble impose de s'interroger sur les manières de préparer les réponses des villes aux défis de phénomènes difficilement prévisibles, brutaux dans leur déclenchement, à cinétique rapide et pour lesquels les populations mais aussi les acteurs en charge d'assurer la sécurité et la sûreté des populations et des territoires peuvent rapidement être pris au dépourvu. Les enjeux étant de penser le niveau opératoire de la résilience non plus uniquement au niveau technique mais aussi au niveau social et territorial, en prenant en compte les vulnérabilités et les capacités des sociétés à engager le cycle de la résilience : pour mieux se préparer et résister, faire face, reconstruire et se reconstruire.

Pour ce faire, nous présentons dans une première partie les rares modèles pouvant être aujourd'hui mobilisés pour mettre en action la résilience territoriale. Puis, dans une seconde partie, en nous appuyant sur les travaux de nos prédécesseurs, nous proposons le modèle CREAA, un cadre opérationnel d'aide à la construction de villes résilientes basé sur quatre modules visant à se préparer, résister, apprendre et rétablir des conditions normales de fonctionnement.

\section{Quelques rares modèles de résilience}

Depuis l'émergence du concept de résilience dans les domaines du changement climatique, des risques et catastrophes, quelques rares modèles d'analyse de la résilience territoriale ont été proposés par la communauté scientifique. Nous avons sélectionné ici quatre modèles susceptibles de répondre à notre questionnement, celui d'identifier un modèle opérationnel, qui puisse aussi bien être utilisé par la communauté scientifique que par des acteurs institutionnels, et qui permette de lancer une réflexion sur la façon de construire un territoire résilient face à une menace, quelle que soit son origine (inondation, séisme, épidémie, accident technologique, ou encore attentat, etc.). 
Dans la littérature scientifique, nous avons ainsi identifié des modèles génériques et stylisés de résilience construits dans une approche systémique, comme celui de Panarchy [GUN 02] ou du Cycle Adaptatif [WAL 04], et des modèles plus opérationnels, comme le Resilience Assessment Framework (RAF) [RES 10] ou le Spatial Decision Support (DS3) [BAL 13]. Il ne s'agit pas ici de présenter ces derniers en détail mais davantage d'identifier leurs intérêts et limites au regard de notre problématique de recherche, à savoir la mise en action de la résilience territoriale et sociétale.

\subsection{Des modèles stylisés issus des sciences "dures"}

Nous ne reviendrons pas ici sur les différentes définitions qui ont pu être données de la résilience en sciences des matériaux, en écologie, psychologie, économie, ou géographie des risques. Des analyses de leurs ressemblances ou divergences ont en effet été réalisées et publiées [ION 16; CHA 14; REG 15'; PRO 13]. Nous nous intéressons davantage aux cadres méthodologiques et modèles conceptuels ayant été mobilisés ou non par les communautés scientifique et opérationnelle.

La résilience systémique est interprétée dans les modèles stylisés du cycle adaptatif [WAL 04] et de Panarchy [GUN 02]. Ces modèles, illustrés par la figure 1 décrivent les dynamiques d'un système (par exemple naturel, humain, territorial, socio-écosystème) exposé à une perturbation. Le cycle adaptatif est un modèle de transition entre différents états qui met bien en lumière le fait que le système se renouvelle et se réorganise à la suite d'un choc. Ce modèle comprend quatre phases qui correspondent aux différentes trajectoires par lesquelles le système étudié transite : tout d'abord la croissance du système (r), puis sa conservation $(K)$, ensuite son effondrement $(\varphi)$ et enfin sa réorganisation, son renouveau $(\alpha)$. La résilience correspond ainsi à la capacité du système à passer d'un équilibre à un autre à travers ces quatre phases [CHA 14].

Toutefois, l'étude des socio-écosystèmes ne peut se faire sans regarder la manière dont ils sont gouvernés à différentes échelles. Le modèle de Panarchy, construit sur la même logique que celui du cycle adaptatif, propose une représentation stylisée pour comprendre les transformations multi niveaux opérant au sein d'un système. Ce modèle inscrit la réflexion dans une perspective multiscalaire qui permet par exemple de représenter les différents acteurs institutionnels engagés à différentes échelles (locale, régionale, nationale, voir internationale) dans la gestion des risques d'inondation [PIG 17]. De même, à la suite d'un choc, la disparition d'un sous-système peut révéler et nourrir la capacité de résilience d'un méta-système. Pour donner un exemple, à Paris, le réseau de métro (méta-système) devrait maintenir son service malgré la non-reconstruction d'une station (sous-système) détruite par un attentat ou une inondation [REG 12].

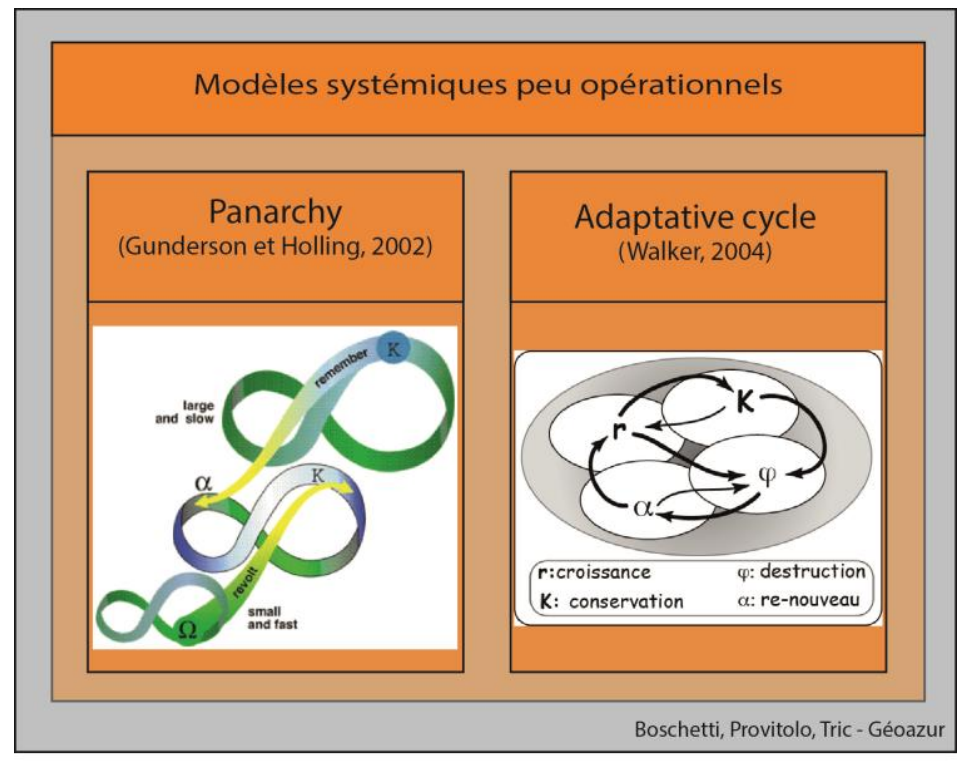

Figure 1. Modèles systémiques d'analyse de la résilience peu opérationnels 
Dans la réalité, ces modèles sont trop rarement mobilisés par les chercheurs et peu connus, voire méconnus des acteurs institutionnels ou opérationnels. Deux raisons peuvent être avancées. Tout d'abord, l'anticipation des crises et donc la mise en place de stratégies, de réponses résilientes est souvent vécue comme une course contre le temps [ROB 10]. Elle entre en compétition avec d'autres priorités identifiées comme plus urgentes car s'inscrivant sur du temps court (par ex. la gestion quotidienne d'aléas mineurs) et révélant des actions plus visibles sur le territoire (par ex. la construction ou le renforcement d'ouvrages de défense). La deuxième en revanche découle directement des modèles proposés. Outre la barrière linguistique, qui fait que ces deux modèles stylisés n'ont pas dépassé les frontières, ces méthodes sont difficiles à mettre en œuvre car elles utilisent un vocabulaire complexe (Panarchy, bifurcation), sont organisées par des phases trop globales et trop éloignées des réflexions locales (par ex. analyser les phases de croissance, de réorganisation des territoires dans les modèles du Cycle adaptatif et de Panarchy) et n'identifient pas les mécanismes de résilience.

Les modèles de Panarchy et du Cycle Adaptatif sont donc peu adaptés à une mise en application réelle, et à une opérationnalisation permettant de construire la résilience d'un territoire.

\subsection{Des modèles à portée opérationnelle}

Plus récemment, des modèles à portée opérationnelle de résilience des individus, des populations ou des territoires ont été développés par les Sciences Humaines et Sociales (la psychologie, l'économie, la géographie, etc.). Deux d'entre eux ont été conçus pour répondre à cette problématique de construction de villes résilientes (figure 2) : le modèle Resilience Assessment Framework (RAF), proposé par le groupe de chercheurs de Resilience Alliance [RES 10], pensé pour les socio-écosystèmes, et le modèle Spatial Decision Support System (DS3) [BAL 13, SER 11], conçu pour étudier la résilience urbaine.

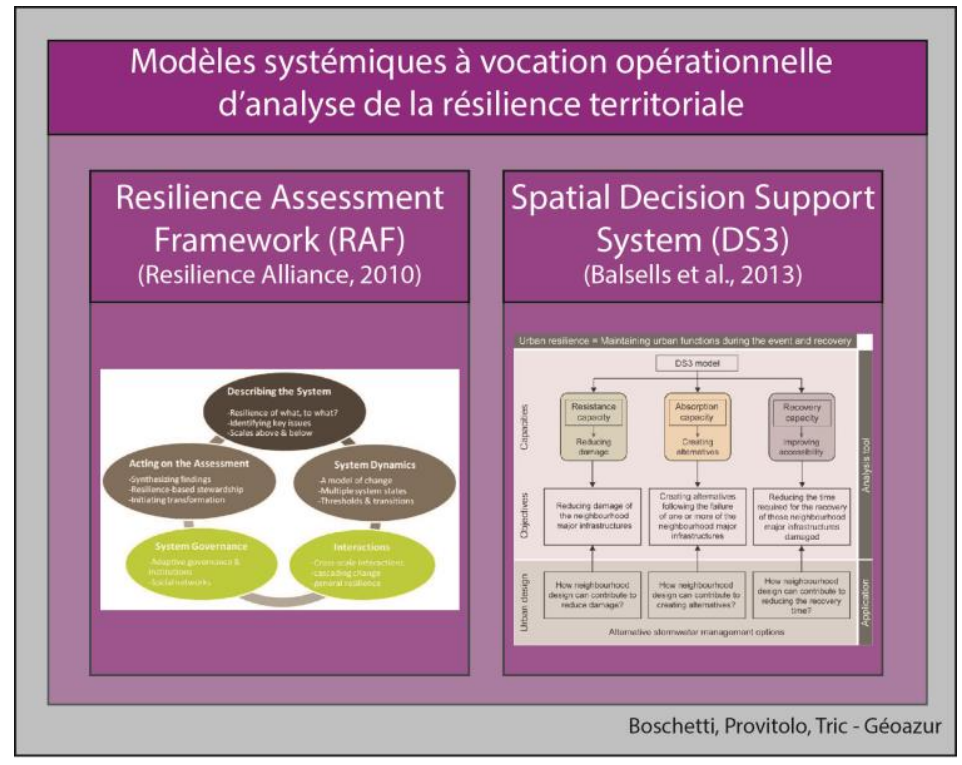

Figure 2. Modèles systémiques à vocation opérationnelle d'analyse de la résilience territoriale

Le modèle RAF est construit autour de cinq modules visant respectivement à décrire le système (module 1), en comprendre ses dynamiques spatio-temporelles (module 2), afin d'identifier les interactions entre différents enjeux territoriaux et les possible effets de dominos (module 3). Des outils spatiaux d'aide à la décision peuvent être réalisés pour mener à bien ces études. Les deux derniers modules visent à évaluer la gouvernance d'un territoire (module 4), afin d'agir sur l'évaluation de la résilience du système (module 5). Un workbook, construit sous la forme d'un guide pratique permettant d'appliquer le modèle proposé, vient expliciter la méthodologie et guide ainsi le lecteur pas à pas dans l'analyse de la résilience des socio-écosystèmes. Sa mise en œuvre nécessite toutefois d'avoir à disposition de nombreuses données aussi bien qualitatives que quantitatives. De plus, une fois encore, le vocabulaire utilisé n'est pas forcément accessible à des non spécialistes. 
Le modèle DS3 propose de dépasser ces limites. Il a été conçu pour analyser la résilience d'un territoire urbain soumis au risque d'inondation, avec une application faite à l'échelle du quartier. Cette échelle d'analyse correspond à celle utilisée pour les réflexions d'aménagement urbain liées aux problématiques d'inondations [SER 12]. Pour se faire, les auteurs se focalisent sur la dimension physique du système urbain, et plus précisément sur les réseaux, qualifiés d'Infrastructures Critiques (IC). Ces IC jouent un double rôle pour la vulnérabilité du territoire: elles sont à la fois des entités à la vulnérabilité intrinsèque, et à la fois des entités propagatrices de vulnérabilité [VIG 06; DÉG 02]. Ce modèle permet ainsi une analyse fine de la zone d'étude choisie, via des méthodes efficaces qui ont déjà fait leurs preuves (théorie des graphes et analyse spatiale via un système d'information géographique).

Il est important de souligner que le modèle DS3 ne vise pas à fournir une évaluation quantitative mais plutôt une analyse qualitative de l'état de résilience d'un quartier inondé ou inondable. Ce modèle conceptuel fournit également un cadre de discussion permettant aux acteurs publics et privés de coconstruire cette résilience à l'échelle infra-urbaine, en portant une attention particulière à la dimension physique du quartier urbain [PRO 17].

Bien que pertinent, il ne répond que partiellement à notre problématique, qui est d'avoir une vision plus élargie des territoires urbains en prenant en compte leurs dimensions physique, sociale, économique et fonctionnelle. Ces modèles (figure 1 et figure 2) ont tous été conçus selon une logique systémique, permettant une prise en compte aussi bien des échelles temporelles que des échelles spatiales. L'une de leur limite est d'être difficilement applicables car trop génériques et trop déconnectés du territoire (Panarchy et Cycle Adaptatif), ou opérationnels mais pensés pour une seule dimension du système (Resilience Assessment Framework et DS3). Notre problématique étant d'obtenir un modèle de résilience opérationnel et applicable par les acteurs, les différents modèles exposés précédemment ne satisfont pas pleinement l'ensemble de ces conditions. C'est pourquoi, en nous appuyant sur les travaux de nos prédécesseurs, nous proposons un nouveau modèle, le modèle CREAA.

\section{Le modèle CREAA}

Le modèle CREAA (figure 3) a été spécifiquement pensé pour construire la résilience des territoires aux risques d'inondations. Trois cibles majeures sont retenues pour promouvoir la résilience des villes (figure 3): les populations et les entreprises, les réseaux durs (par ex. infrastructures de communication, énergie, assainissement etc.) et soft (plus particulièrement les acteurs institutionnels et opérationnels, formels et informels), et enfin les déchets. Ces cibles constituent le point de départ de notre raisonnement et dans le cadre de cette étude, correspondent aux points clés du territoire sur lesquels travailler.

Le modèle CREAA est un modèle systémique qui a été construit à partir d'un terrain et d'un scénario de risque, la Côte d'Azur, soumise à l'aléa inondation. Il a cependant pour ambition d'avoir une méthodologie reproductible à tous types de territoires, qu'ils soient soumis au même type d'aléa que celui présenté, c'est-à-dire l'inondation, ou à d'autres types d'aléas (sismique, climatique, technologique, etc.). C'est pourquoi nous utiliserons des exemples divers pour illustrer cette étude.

L'objectif principal est de construire et de mettre en place les résiliences des territoires et des sociétés. Ce modèle n'a donc pour but ni de quantifier cette dernière, ni de fournir une liste d'indicateurs, comme le propose par exemple S. Cutter [CUT 10]. Ces derniers quantifient la résilience, via des indicateurs balayant divers aspects, comme la résilience sociétale (âge, accès au transport, etc.), la résilience économique (pourcentage de femmes actives, chômage, etc.), la résilience institutionnelle (nombre de déclaration de sinistres payés, nombre d'états et de circonscriptions, etc.), la résilience des infrastructures (pourcentage de logement locatif vacant, nombre de lits d'hôpitaux pour 10000 
habitants, etc.), et le capital communautaire (migration, nombre d'adhérents religieux pour 10000 habitants, etc.).

Bien qu'intéressante, cette mise en indice peut parfois apparaitre difficile à mettre en place de par le choix des indicateurs et par l'accès à la donnée. Elle peut également être limitante dans les indicateurs pris en compte. De plus, certains éléments sont difficiles à saisir sous forme d'indices. On pense notamment aux actions permettant d'accroître la culture du risque ou encore à celles à mettre en place après une catastrophe. Il faut également faire attention à ne pas tomber dans une analyse de vulnérabilité « camouflée » en analyse de la résilience.

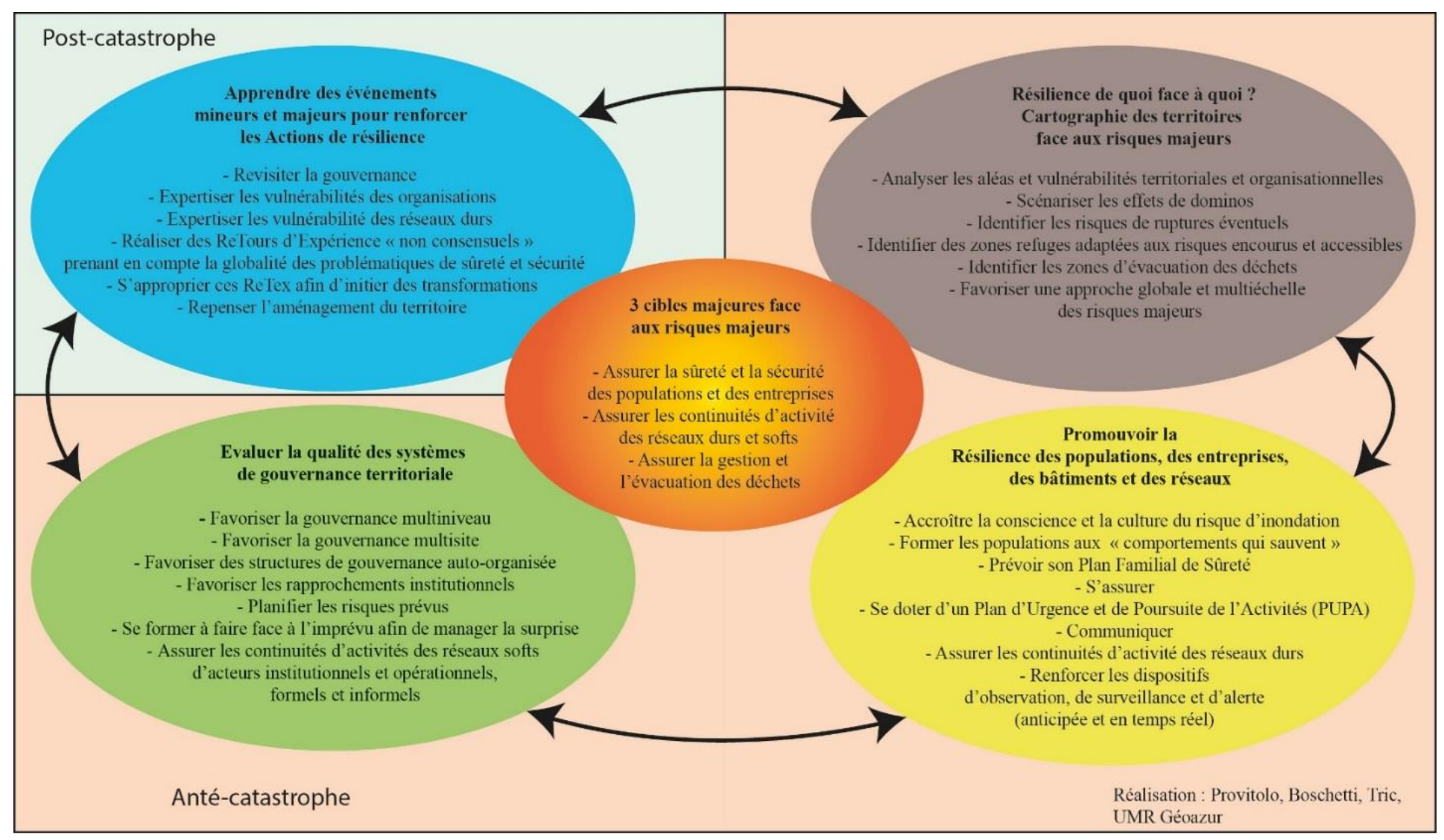

Figure 3. Le modèle CREAA : un cadre méthodologique à portée opérationnelle pour promouvoir des territoires et des sociétés résiliences

La mise en place d'indicateurs nous apparait comme une solution possible d'analyse de la résilience, mais qui passe dans un premier temps par la construction de la résilience et par la mise en place d'outils à destination des opérationnels. Nous nous focalisons donc dans ce papier sur la construction de cette dernière, via la mise en place d'un modèle conceptuel, le modèle CREAA.

Pour mettre en action la résilience des territoires, deux temporalités doivent être considérées comme des cycles interconnectés : l'avant et l'après événement. Ainsi, pour chacune de ces temporalités, différentes capacités peuvent être mobilisées [REG 15] :

- L'avant événement : avec la capacité d'anticipation et la capacité d'apprentissage ;

- L'après le choc : avec la capacité à récupérer et à se reconstruire, à revenir à un état d'équilibre, et à s'adapter.

Le fait d'agir sur ces deux temporalités permet de préparer le territoire, les populations, les acteurs institutionnels, etc., en amont et en aval d'un événement. Il n'y a que durant l'événement que l'on peut vraiment évaluer la résilience [RIC 03] et voir si les actions préalablement déployées répondent à la complexité d'une menace ou d'une crise. Bien que tirée de l'économie, cette temporalité de la construction de la résilience s'applique également au domaine des risques et des catastrophes. Si un 
travail est fait par une collectivité ou une société sur un territoire, de manière à préparer la population et l'«armer» le mieux possible face aux différentes menaces auxquelles elle est exposée (par différentes actions comme l'information ou encore la mise en place de documents règlementaires). Il en va de même avec la phase qui vient après un événement et qui permet d'apprendre de ses erreurs, et mettre en place les mesures nécessaires pour être davantage préparé au prochain événement.

Le modèle CREAA est structuré en quatre parties. Chacune s'intéresse à un élément particulier, que nous allons détailler par la suite :

- Cartographier les territoires face aux inondations (C)

- Promouvoir la Résilience des populations, des entreprises, des bâtiments et des réseaux $(\mathrm{R})$

- Evaluer la qualité des systèmes de gouvernance territoriale (E)

- Apprendre des événements mineurs et majeurs pour renforcer les Actions de résilience (AA)

\subsection{Cartographier les territoires face aux inondations (C)}

Le premier module (figure 4), intitulé «Cartographie des territoires face aux inondations (C)», consiste en la réalisation d'un bilan des connaissances de la zone d'étude. Sont ainsi pris en compte les analyses d'aléas et de vulnérabilités, permettant une connaissance du risque et des enjeux majeurs sur le territoire étudié. Il est primordial d'avoir une connaissance approfondie du territoire sur lequel on travaille, pour en connaître les particularités, les points forts et les points faibles. Cette connaisse est indispensable à la mise en place d'une étude portant sur la résilience territoriale.

A partir des aléas et du territoire d'étude, il convient de scénariser les effets dominos, c'est-à-dire les différents aléas en chaine susceptibles de se produire. Ces aléas peuvent appartenir au même domaine, le naturel par exemple pour un enchaînement de type séisme-tsunami-glissement de terrain, ou à des domaines distincts, on parle d'effets dominos de type Nat-Tech. La catastrophe de Fukushima s'inscrit par exemple dans cette dernière catégorie.

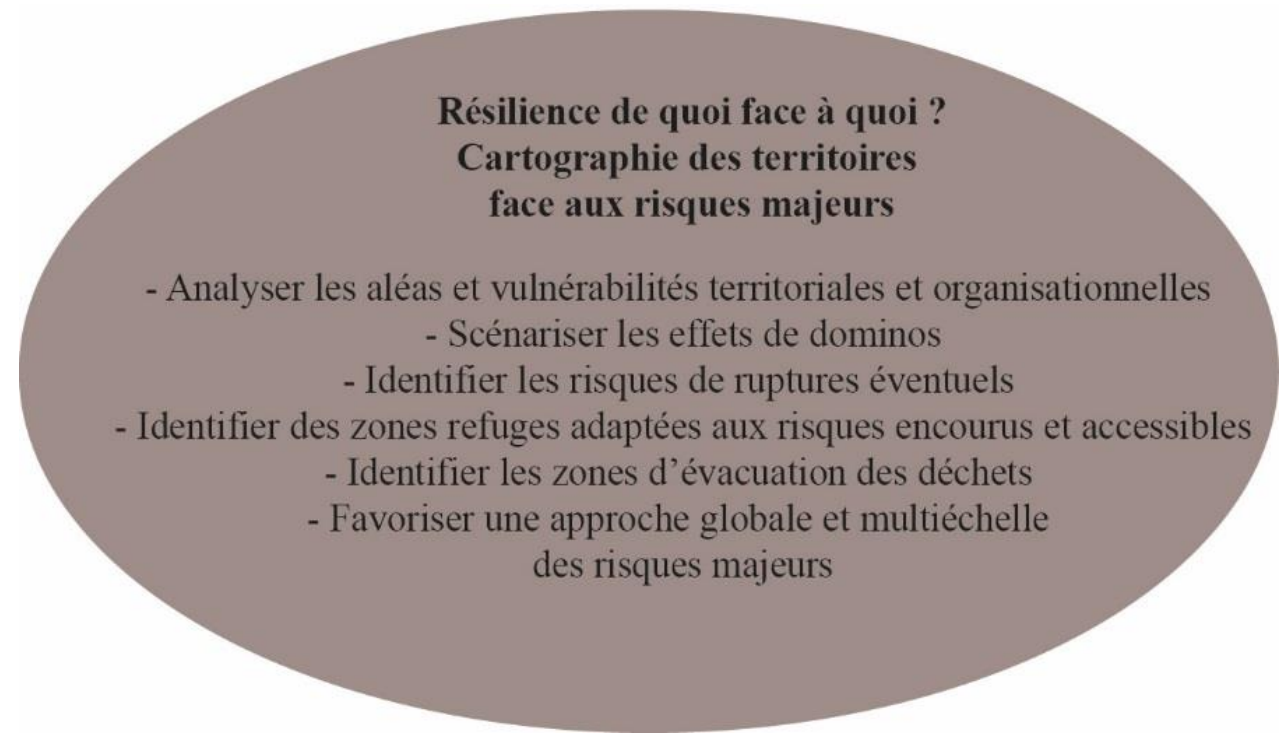

Figure 4. Module $C$ : Cartographie des territoires face aux inondations

Doivent également être pris en compte les points clés du territoire, qui peuvent ressortir des analyses de vulnérabilité qui auront été réalisées au préalable. Il peut s'agir de nœuds d'axe routier, qui en cas de rupture vont fragiliser l'ensemble du réseau, comme par exemple un échangeur routier ou autoroutier, qui va empêcher soit l'accès à cet axe, soit inversement, en empêcher la sortie, et rendre difficile la circulation, principalement des secours, mais aussi des populations. 
En nous appuyant sur la connaissance du fonctionnement territorial et sur le type de menace auquel il faut faire face, il convient alors de déterminer et de localiser des zones refuges pour assurer la mise à l'abri, la sécurité et la sûreté des citoyens. Une zone refuge ne doit pas être déterminée de la même manière selon que l'on fait face à une inondation, un séisme ou une menace terroriste. Il est donc important d'adapter les zones refuges aux spécificités de la menace. Ces zones peuvent être i) prédéfinies et connues de la population [où indiquées par une signalisation particulière, comme c'est le cas sur différents littoraux exposés au risque de tsunami, par exemple en Thaïlande ou le long de la côte ouest des Etats-Unis], ii) déterminées sur le moment en fonction de la connaissance du terrain, de son accessibilité et de la capacité de tout un chacun d'analyser rapidement une situation imprévue et de prendre une décision.

Lorsque ces zones refuges sont déterminées au préalable par les autorités, un ensemble de paramètres doit être pris en considération, allant de la capacité d'accueil, aux capacités matérielles, en passant par l'accessibilité en temps de crise (COI). Une préparation en amont facilite la gestion et la rapidité d'action car les autorités savent où orienter la population sinistrée. Lors des inondations qui ont frappé les Alpes Maritimes en octobre 2015, l'une des municipalités avait prévu des sites refuges afin de mettre à l'abri les sinistrés en cas d'événement de ce type. Une réflexion avait donc été lancée en amont. Or, il s'est avéré que lors de sa mise en application, certains lieux choisis ne pouvaient être utilisés, car non accessibles du fait de l'inondation.

Les événements de grande ampleur sont généralement générateurs de nombreux dégâts matériels, demandant une évacuation de quantités de gravats et de déchets. Une réflexion en amont de leur évacuation et de leur traitement est primordiale, afin de savoir comment les évacuer, où les stocker, comment les traiter, etc. Il s'agit dans un premier temps de "libérer » la ville afin d'effectuer les recherches de survivants plus facilement. Dans un deuxième temps, la reconstruction ne peut être lancée qu'une fois l'ensemble des gravats et des déchets évacués. Après le séisme de Christchurch de 2011 en Nouvelle-Zélande, une grande partie de la ville a été détruite, il a donc fallu commencer par évacuer les gravats pour ensuite pouvoir lancer la reconstruction.

Une approche globale et multi-échelle (spatiale et temporelle) est donc indispensable pour lancer les réflexions sur la construction de la résilience d'un territoire. D'autres actions portant sur les populations, les entreprises, les bâtiments et les réseaux sont à mettre en place.

\subsection{Promouvoir la résilience des populations, des entreprises, des bâtiments et des réseaux} (R)

Ce module (figure 5) se focalise sur les cibles majeures, qui sont la population, les entreprises, les bâtiments et les réseaux.

Promouvoir la

Résilience des populations, des entreprises, des bâtiments et des réseaux

- Accroître la conscience et la culture du risque d'inondation

- Former les populations aux « comportements qui sauvent»

- Prévoir son Plan Familial de Sûreté

- S'assurer

- Se doter d'un Plan d'Urgence et de Poursuite de l'Activités (PUPA)

- Communiquer

- Assurer les continuités d'activité des réseaux durs

- Renforcer les dispositifs

d'observation, de surveillance et d'alerte

(anticipée et en temps réel)

Figure 5. Module $R$ : Promouvoir la Résilience des populations, des entreprises, des bâtiments et des réseaux 
Promouvoir la résilience auprès des populations demande un travail en collaboration avec ces dernières afin de les sensibiliser aux problématiques des risques et de catastrophes. Pour ce faire, des campagnes d'information et de formation doivent être lancées avec pour objectif l'accroissement de leurs connaissances sur les différents risques susceptibles d'affecter leurs lieux de vie. Il est malheureusement souvent nécessaire qu'une catastrophe se produise pour qu'une prise de conscience ait lieu (aussi bien du côté des élus que du côté de la population) et pour que des actions soient mises en place. C'est le cas par exemple de certaines communes et communautés de communes des AlpesMaritimes qui, suite aux inondations meurtrières d'octobre 2015, ont réalisé des réunions d'information pour la population.

Informer la population des gestes qui sauvent, ou inversement qui peuvent mettre en danger permet de rendre chaque citoyen acteur de sa propre sécurité [PRO 15]. Il peut s'agir d'actions simples, comme de fermer les bouteilles de gaz et les éloigner des habitations en cas de feux de forêt à proximité d'une habitation, ou des actions qui vont à l'encontre de l'instinct humain comme le fait de ne pas aller chercher ses enfants à l'école en cas d'orage violent. Ces comportements sont malheureusement souvent la cause de décès lors d'événements exceptionnels. Ce fût le cas lors des inondations d'octobre 2015 dans les Alpes-Maritimes, puisque la plupart des décès sont dus à une mauvaise connaissance du risque et à une mise en danger non évaluée. Certains habitants d'immeubles dont les garages étaient souvent inondés, avaient pris pour habitude de s'avertir les uns les autres quand le phénomène se produisait, pour aller ensemble sortir leurs voitures respectives. Ces personnes n'avaient pas conscience que par ces actions et la ritualisation de ces dernières, elles se mettaient involontairement en danger, et qu'en cas d'événement plus important qu'à la normale, ces garages en sous-sol se transformaient en véritables pièges dont il était impossible de ressortir. Il est donc indispensable de travailler sur cet aspect, afin de sensibiliser et surtout former la population, afin qu'elle soit en mesure de se mettre seule en sécurité en cas d'événement, ayant fait l'objet d'une alerte ou non. Cette mise en sécurité permet dans un premier temps de sauver des vies, mais aussi de ne pas mobiliser et/ou mettre en danger les acteurs en charge de la protection civile.

Un travail de sensibilisation de la population doit ainsi être mené par les municipalités. Les mairies ayant un Plan de Prévention des Risques (PPR) sont d'ailleurs dans l'obligation de se doter d'un DICRIM (Document d'Information Communal sur les Risques Majeurs). Toutefois ces outils restent encore peu appropriés par la population. Certaines communes voient via ce document un réel moyen de communication auprès de la population. La ville de Nice par exemple édite un DICRIM à destination de l'ensemble des citoyens, mais aussi un DICRIM jeune, pensé pour un jeune public. Il est ainsi simplifié, utilisant des schémas et des mots simples, mais le message de fond est conservé. La création de ce double document permet de toucher un large public et de commencer la sensibilisation dès le plus jeune âge.

La transmission d'information à la population doit permettre de mettre en avant les différents outils existants, tel que le Plan Familial de Mise en Sureté. Celui-ci permet d'aborder la question des risques majeurs, de la commune de vie, au sein de la famille et de réfléchir à ce qu'il convient de faire en cas d'événement majeur. Il aide également à organiser son autonomie durant la phase critique de l'événement, grâce à la constitution d'un kit d'urgence, comprenant des éléments allant de la bouteille d'eau, à la radio, au nécessaire d'hygiène.

L'assurance des biens, immobiliers, moyens de transports (voitures, motos, etc.), ou encore d'une entreprise, permet aux sinistrés d'avoir le moins de pertes économiques possible. Cette indemnisation va cependant être fonction du type d'assurance souscrite, mais va également dépendre du vote (ou non) de l'arrêté de catastrophe naturelle (Arrêté CatNat) en conseil des Ministres. Si l'on reprend l'exemple des inondations des Alpes-Maritimes de 2015, deux jours après les inondations, des camions appartenant à de grandes compagnies d'assurance sont venus s'installer sur des parkings de proximité, permettant aux sinistrés d'obtenir des renseignements, de remplir des dossiers de sinistre afin de lancer les démarches rapidement. 
Les institutions et les entreprises doivent quant à elles se doter d'un PUPA (Plan d'Urgence et de Poursuite d'Activités) qui a pour but de réfléchir en amont à des solutions de repli leur permettant de continuer de fonctionner en cas de crise. Il peut s'agir par exemple de locaux où se réfugier et où poursuivre l'activité, comme une deuxième cellule de crise pour la commune dans le cas où la première venait à être défaillante.

Comme nous l'avons vu précédemment, les réseaux « durs » font partie des infrastructures majeures d'un territoire, il faut donc assurer la continuité de ces réseaux. Il s'agit généralement des axes de communications, d'eau, d'électricité, etc., ces derniers doivent donc être fonctionnels en permanence. En cas d'incident sur un point d'un des réseaux il faut s'assurer que ce dernier continue à fonctionner, même en mode dégradé, et que l'ensemble du réseau ne devienne pas défaillant.

Les aléas présentent un réel danger pour l'homme, c'est pourquoi de nombreux scientifiques travaillent sur la compréhension de leur mécanisme et sur différents dispositifs permettant de les observer et de les surveiller. Lorsque ceux-ci sont connus et compris, il est possible de mettre en place des systèmes d'alerte, permettant de passer de l'observation et de la surveillance scientifique à l'information de la population. C'est le cas par exemple avec les alertes météorologiques lancées régulièrement par Météo France. Ces dernières se basent sur des bulletins météorologiques en temps réel et sur des modèles informatiques qui permettent de prévoir (avec une marge d'erreur) l'évolution des différentes perturbations. Lorsqu'un certain seuil est franchi, les municipalités possiblement impactées sont prévenues et les alertes (jaune, orange ou rouge) sont lancées.

Il est donc impossible de construire la résilience sans inclure les éléments structurants d'un territoire, qu'il s'agisse d'éléments vivants, comme la population, de composantes dynamiques comme les entreprises ou les réseaux, ou encore d'éléments statiques comme les bâtiments. Il faut cependant ajouter à cela la gouvernance territoriale.

\subsection{Evaluer la qualité des systèmes de gouvernance territoriale (E)}

Ce troisième module (figure 6) s'articule autour de la gouvernance. Elle est définie par John Pitsey [PIT 10] comme recouvrant «à la fois l'éthique en politique, le contrôle des représentants politiques, la réforme des institutions internationales, les accords public-privé, la réforme du management des entreprises publiques, etc.». Ce à quoi il ajoute qu'elle est définie par sa capacité à former une décision et non pas à partir du type de procédure qu'elle mobilise.

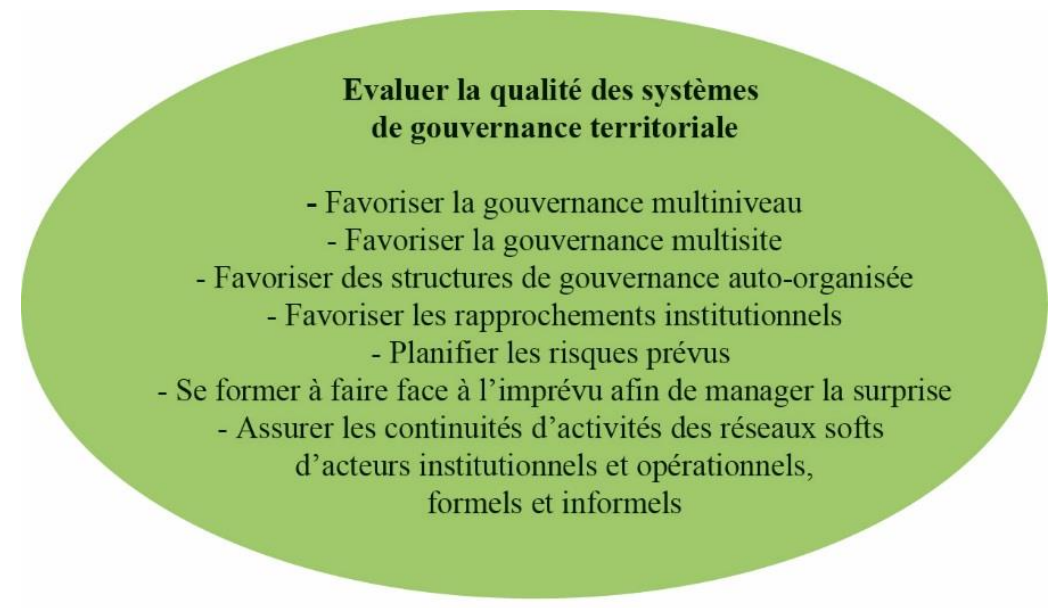

Figure 6. Module E : Evaluer la qualité des systèmes de gouvernance territoriale

Le système institutionnel français est relativement complexe et présente de nombreux échelons d'action. C'est pourquoi, il est nécessaire de développer une gouvernance multi-niveau et multi-site. L'auto-organisation doit également être favorisée, les structures peuvent ainsi disposer d'une liberté 
d'action en cas de crise, leur permettant d'agir rapidement et de manière efficace, ce qui est déjà le cas lorsqu'un événement se produit à l'échelle communale. La centralisation de la décision et la prise en charge de la gestion de crise par un pouvoir décisionnel supérieur est tout de même souhaitable lorsqu'un événement impacte un grand territoire, afin d'avoir une coordination des actions déployées sur le terrain. Il est toutefois souhaitable de laisser une liberté d'action aux institutions locales lors de l'événement et dans la première partie de la gestion de crise, car elles connaissent leur terrain et les ressources dont elles disposent.

Ces institutions sont nombreuses, et ne sont pas nécessairement en lien les unes avec les autres. Il est donc primordial d'encourager ces rapprochements. Une gouvernance structurée, préparée et favorisant les liens entre les différentes institutions pourrait améliorer la gestion de la crise. Lorsqu'une institution est organisée, que chacun connait sa fonction et son rôle en cas d'événement, lorsqu'une crise vient à se produire, elle devrait être plus facilement gérée. Si l'on prend comme exemple les événements (inondation de la Seine en 2016, feux de forêt dans le sud de la France au cours de l'été 2017, etc.) qui se sont produits récemment en France, ces derniers ont été globalement bien gérés avec la mise en place d'actions nécessaires à la mise en sécurité de la population. Si à l'inverse nous prenons l'exemple de catastrophes se produisant dans d'autres pays, où l'État n'est pas autant préparé et organisé, les dégâts peuvent être bien plus importants, voire catastrophiques. C'est le cas par exemple en Haïti, petit pays insulaire soumis à de nombreuses menaces qui, de par la pauvreté de ses habitants, l'absence de construction parasismique et l'instabilité du gouvernement haïtien, le pays se remet à peine du séisme destructeur de 2010, et doit aujourd'hui faire face aux destructions occasionnées par l'ouragan Irma, qui s'est produit début septembre 2017.

Les différentes institutions françaises ont l'obligation d'intégrer les risques présents sur leur territoire dans les différents plans (d'aménagement, de gestion de crise, etc.) qu'elles doivent constituer. Les risques dits « prévus » correspondent à ceux qui frappent régulièrement le territoire, et qui sont donc connus, maîtrisés, et que l'on est en capacité de prévoir. Ils font également partie intégrante des plans mis en place. C'est le cas par exemple du risque d'inondation, et plus particulièrement les inondations «lentes », qui sont prévisibles de par leur dynamique temporelle, et contre lesquelles il est possible d'agir. Ce fut le cas par exemple lors de la crue de la Seine en 2016, qui a été relativement bien gérée au niveau de la capitale, limitant les inondations dans Paris, grâce à la mise en place et à l'utilisation de lacs-réservoirs en amont [SEI 16], qui permettent de stocker une grande quantité d'eau et ainsi de réduire les débits de la Seine à Paris.

En opposition à ces risques «prévus », il est primordial d'arriver à former les institutions et les populations à l'imprévu. Connaître les actions et les réactions à avoir en cas d'événement inattendu aide à mieux les appréhender. Agir efficacement contre un événement attendu peut s'avérer difficile, et cela se complexifie encore davantage lorsqu'il n'y a pas ou peu de préparation. Prenons l'exemple des inondations, de type cévenol, caractérisées par de grandes quantités d'eau qui s'abattent sur un territoire en quelques heures. Ces épisodes sont plus compliqués à prédire et de par la rapidité de l'événement, les acteurs institutionnels ont peu de temps pour réagir. Il en va de même pour les populations, qui doivent adapter leur comportement à ces types de menaces afin de ne pas se mettre inutilement en danger.

Les institutions ayant connaissance de l'importance de la préparation à l'imprévu, des actions sont mises en place pour entrainer les acteurs, comme par exemple les exercices Richter. Ces derniers sont des exercices de gestion de crise, organisés par la Direction Générale de la Sécurité Civile et de la Gestion des Crises (DGSCGC) en collaboration avec les préfectures. Ces exercices se basent principalement sur le risque sismique et les risques associés comme les glissements de terrain ou les tsunamis. L'objectif étant de simuler une crise, grâce à un scénario prévu en amont (non connu des acteurs institutionnels), et d'injecter petit à petit des actions sur le territoire afin d'observer la gestion qui en est faite en situation de stress. Après coup, il y a un retour d'expérience « à chaud », c'est-à-dire directement après la fin de l'exercice, permettant d'avoir des retours immédiats sur ce qui a fonctionné 
ou ce qui n'a pas fonctionné, ainsi que sur le ressenti de chacun. Puis un second retour d'expérience est organisé quelques mois plus tard, laissant le temps à chaque institution de faire un point plus en profondeur, et de préciser ce sur quoi cela l'a amené à réfléchir, voir sur les actions qui ont commencé à être mises en place.

L'intégration de la gouvernance à ce modèle de construction de la résilience, vient compléter les autres composantes du systèmes (population, entreprises, réseaux, etc.). Cet ensemble s'intègre dans l'ante-événement, en agissant en amont d'une crise, on tente d'améliorer le moment critique d'une crise, afin que cette dernière se déroule de manière la moins catastrophique possible. Vient s'ajouter à cela un module centré sur le post-crise.

\subsection{Apprendre des événements mineurs et majeurs pour renforcer les Actions de résilience $(A A)$}

Cette dernière phase (figure 7), intitulée «Apprendre des événements mineurs et majeurs pour renforcer les Actions de résilience », sort de la temporalité des modules précédents, c'est à dire de l'ante-événement, pour entrer dans le post-crise. Il s'agit ici de faire un bilan de ce qui s'est produit afin de renforcer et améliorer les différentes actions existantes. Il convient de conserver ce qui a fonctionné et de travailler sur ce qui au contraire a présenté des faiblesses. Il est important de souligner que cette étape doit être réalisée pour tous les événements, qu'ils soient mineurs ou majeurs, qui ont marqué le territoire, car on apprend également des petits événements.

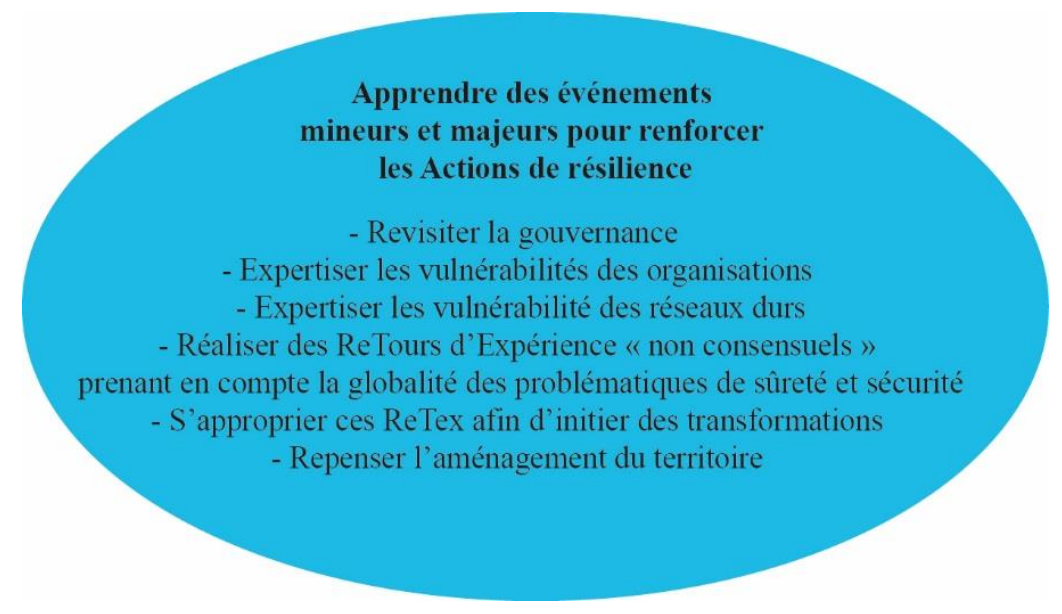

Figure 7. Module AA : Apprendre des événements mineurs et majeurs pour renforcer les Actions de résilience

Si nous reprenons le cas des inondations, souvent, pour la réalisation des ouvrages techniques et des documents officiels (de type PPR ou PCS), sont pris comme événement de référence les événements connus. Or, ces événements d'intensité exceptionnelle, se caractérisent par une faible probabilité d'occurrence, ce qui en rend l'observation réelle difficile. Cependant, des événements de plus faible intensité viennent à se produire de manière plus ou moins régulière, pouvant causer ou non des dégâts. Ils sont donc de bons tests de mise en application des outils à disposition des collectivités pour justement les tester et en fonction du déroulement apporter les modifications nécessaires. Ces derniers permettant de se préparer à un événement de plus grande ampleur. Il faut tout de même relativiser ces propos, si ces derniers présentent des problèmes de mise en application en cas d'événements mineurs, il est fort probable qu'en cas d'épisode extrême, les dysfonctionnements de ces outils soient encore plus importants. A l'inverse, si la gestion de l'événement s'est bien déroulée, cela ne signifie pas automatiquement que tout se passera bien en cas d'événement d'intensité supérieure.

Cette étape passe par une série d'expertises et de bilans. Il peut s'agir d'un Retour d'Expérience (ReTex) général à l'ensemble de l'événement, ou alors à une série de ReTex plus spécialisés sur des points clés ou concernant des organisations particulières comme par exemple au sein même du SDIS, de la Croix-Rouge, ou encore de la SNCF. Les ReTex sont cruciaux pour bien comprendre le 
déroulement de l'événement dans sa globalité. Ces analyses ont un réel rôle à jouer dans le postévénement et dans la compréhension de ce dernier. C'est pourquoi elles doivent être réalisées par des personnels qui ont été impliqués dans la gestion de la crise. Les ReTex doivent être facilement appropriables par les différents organismes, afin que des actions puissent être mises en place pour rendre encore plus efficaces la prévention et la gestion des risques. Trop souvent, ces ReTex sont réalisés à un échelon haut, et redescendent rarement vers les petites communes (démarche «topdown»), qui ont des difficultés à se remettre de la catastrophe et à mettre en place les actions nécessaires.

Le lien vulnérabilité-résilience est aujourd'hui encore flou [CUT 08, PRO 13]. Il est difficile de savoir où se situe l'un par rapport à l'autre, et comment articuler ces deux notions. Plusieurs visions se retrouvent dans la littérature : i) vulnérabilité et résilience peuvent être vues comme antonymes, ii) la vulnérabilité peut être vue comme une composante de la résilience, iii) elles peuvent également être vues comme un continuum, disposant de liens bien plus complexes, avec la notion de «vulnérabilité résiliençaire » [PRO 15’].

Dans le post événement, il apparait pertinent de réaliser une expertise des vulnérabilités d'éléments clés du système, que sont les organisations et les réseaux « durs ». Celle-ci a pour but de faire un bilan sur le maintien de ces infrastructures critiques, qui en cas de rupture, vont avoir un impact sur l'ensemble du territoire. Il ne s'agit pas ici de réaliser une analyse de vulnérabilité territoriale comme ont pu le proposer D'Ercole et Metzger dans leurs travaux [D'ER 09] portant sur Quito. En revanche, nous gardons la notion d'enjeux majeurs qu'ils proposent, et qu'ils définissent dans cette article de 2009 comme étant « des éléments clés qui permettent à l'ensemble d'un territoire de fonctionner, de se développer et de faire face à une situation d'urgence [...] (et qui) permettent à l'ensemble du territoire métropolitain d'assurer ses fonctions sociales, économiques et politiques en période normale et ses fonctions de secours et d'appui à la population en période de crise». En post-événement il s'agit davantage d'analyser la tenue et la vulnérabilité réelle de ces enjeux majeurs, en réalisant des observations et des études sur le terrain.

L'objectif final étant de repenser l'ensemble de l'aménagement face aux risques qui menacent le territoire, afin d'en réduire les vulnérabilités. Les changements qui vont être opérés à la suite de catastrophes, font suite à des observations faites sur les bâtiments ou les infrastructures, déterminant une vulnérabilité réelle et non plus hypothétique comme lors d'analyse de vulnérabilité territoriale faite en ante-événement. Ces changements vont donc cibler en priorité les enjeux majeurs du territoire qui ont été impactés ou qui pourraient l'être lors d'un événement futur, et par la suite les structures mettant en danger la population, comme par exemple un lotissement construit dans le lit majeur d'une rivière. La composante exposition au risque est donc prise en compte dans ces analyses de vulnérabilité postcrise. Cela prend du temps, et demande parfois des changements en profondeur dans la manière de penser le territoire.

Etant dans un modèle systémique, la temporalité est cyclique, on a donc un cycle avec trois temporalités de la catastrophe (ante-, pendant et post-), la dernière phase devenant la première d'une nouvelle mise en action et transformant le système. Il ne s'agit pas de revenir à l'état du système lors de la phase d'ante-événement, mais à un état amélioré où le système est davantage préparé à une future crise.

Ce modèle ne prend donc pas en compte l'ensemble des temporalités de l'événement, il se concentre sur l'ante- et le post-crise. Il ne constitue donc pas une analyse de résilience à proprement parler, mais a pour objectif de construire cette résilience pour le territoire, la population et les acteurs. 


\section{Conclusion et perspective}

La tendance à la croissance globale du nombre de catastrophes, toute origine confondue, au cours du dernier siècle s'est accompagnée d'une nette décroissance des pertes humaines [DAU 13]. Afin de pérenniser cette trajectoire de décroissance, tout au moins dans les pays développés, la résilience, qu'elle soit individuelle, collective ou sociétale, est questionnée dans son champ opérationnel.

Cet article avait donc pour objectif premier de dresser un rapide état de l'art sur les différentes méthodologies existantes et permettant de réaliser des analyses de résilience.

Nous avons ainsi mis en lumière le fait que pour répondre à notre questionnement, qui est de permettre aux acteurs (individuels, institutionnels et opérationnels) de construire leur résilience ainsi que celle de leur territoire, quatre modèles peuvent être mobilisés. Nous avons proposé de catégoriser ces derniers en deux groupes: les modèles «génériques » et stylisées de Panarchy et du Cycle Adaptatif, et les modèles plus opérationnels de Resilience Alliance et DS3.

Toutefois, face aux limites de ces derniers, à savoir: le fait d'être génériques et trop peu opérationnels, de prendre difficilement en considération l'ensemble des dimensions du système, nous proposons le modèle CREAA. Celui-ci a pour objectif de Cartographier les territoires face aux risques majeurs ; promouvoir la Résilience des populations, des entreprises, des bâtiments et des réseaux ; Evaluer la qualité des systèmes de gouvernance territoriale; Apprendre de événements mineurs et majeurs pour renforcer les Actions de résilience.

Construit dans une logique systémique, ce modèle ambitionne de donner des pistes pour construire la résilience des villes en amont et en aval d'un événement, d'englober l'ensemble des échelles spatiales et des dimensions du système, de se concentrer sur des cibles majeures regroupant et expliquant clairement ce sur quoi va se concentrer l'étude. Ce modèle a la volonté d'être opérationnel et d'aider les institutions et les décideurs dans la construction de leur territoire.

Le modèle CREAA présente également certaines limites qu'il faudra dépasser : la principale étant qu'il n'englobe pas l'ensemble du continuum temporel du système, mais seulement l'ante- et le postévénement ; étant dense et fourni, il demande une connaissance pointue du territoire et la disposition d'un grand nombre d'information et de données sur ce dernier; et enfin il s'agit d'un modèle conceptuel, pensé de manière opérationnelle, mais qui doit encore faire ses preuves lors de sa réelle mise en application sur une zone d'étude soumise à un ou des risques particuliers.

\section{Bibliographie}

[ASC 00] ASCHAN-LEYGONIE C., Vers une analyse de la resilience des systems spatiaux, Espace géographique, tome $29, n^{\circ} 1$, p. 64-77, 2000.

[BAL 13] BALSELLS M., BARROCA B., AMDAL J. R., DIAB Y., BECUE V., SERRE D., Analysing urban resilience through alternative stormwater management options: application of the conceptual Spatial Decision Support System model at the neighbourhood scale, Water Science \& Technology, IWA 68 (11): 2448-2457, doi: 10.2166/wst.2013.527, 2013.

[CHA 14] CHATAIGNIER J.M., Fragilités et résilience, Les nouvelles frontières de la mondialisation, Eds Karthala, 482 p, 2014.

[CUT 08] CUTTER S., A place-based model for understanding community resilience to natural disasters, Global Environmental Changes, 18, 598-606, 2008.

[CUT 10] CUTTER S., BURTON C., EMRICH C., Disaster Resilience Indicators for Benchmarking Baseline Conditions, Journal of Homeland Security and Emergency Management, Vol. 7 : iss. 1, Article 51, 2010.

[D'ER 09] D'ERCOLE R., METZGER P., La vulnérabilité territoriale : une nouvelle approche des risques en milieu urbain, Cybergeo : European Journal of Geography, document 447, http://cybergeo.revues.org/22022, 2009. 
[DAU 13] DAUPHINÉ A., PROVITOLO D., Risques et catastrophes - Observer, spatialiser, comprendre, gérer, A. Colin (2ème édition), Collection U, 412 p, 2013.

[DÉG 02] DÉGARDIN F., Urbanisation et inondations : de l'opposition à la réconciliation, Bulletin de l'Association de géographes français, $\mathrm{n}^{\circ} 1$, p. 91-103, 2002.

[GEN 16] GENIN D., MAZUREK H., La résilience des systèmes socioécologiques : d'une intuition holiste à une difficile conceptualisation et mise en œuvre, Résiliences - Ressemblances dans la diversité, IONESCU S., Odile Jacob, p. 6392, 312 p, 2016.

[GUN 02] GUNDERSON L.H., HOLLING C.S. (eds), Panarchy: Understanding Transformations in Human and Natural Systems, Island Press, Washington, DC. Wilson EO, 2002.

[ION 16] IONESCU S., Résiliences - Ressemblances dans la diversité, Odile Jacob, 312 p, 2016.

[LAG 09] LAGADEC P., La question des Plans, entre points d'appui et pièges stratégiques, Cahier n 40 du département d'économie de l'école polytechnique, 2009.

[MAZ 17] MAZUREK H., Communication Résilience et pratiques de société et de nature, Symposium international, Bucharest, octobre 2017,2017.

[NAT 12] National Research Council, Disaster Resilience: A national Imperative, Washington, DC: The National Academies Press. 2012.

[O'RO 07] O'ROURKE T. D., Critical infrastructure, interdependencies and resilience., The Bridge - The Journal of the National Academy of Science, 37 (1), p 22-31, 2007.

[PIG 17] PIGEON P., Nexus and Disaster Prevention : What Can We Learn from the Genevan Urban Area?, Peri_Urban Areas and Food-Energy-Water Nexus, p 71-79, 2017.

[PIT 10] PITSEY J., Le concept de gouvernance., Revue interdisciplinaire d'études juridiques (Volume 65), 2010.

[PRO 13] PROVITOLO D., Resiliencery vulnerability notion - Looking in another direction in order to study risks and disasters, Resilience and urban risk management, Serre D., Barocca B., Laganier R. (Eds.), CRC Press, Taylor and Francis Group, 2013.

[PRO 15] PROVITOLO D., et al., Les comportements humains en situation de catastrophe : de l'observation à la modélisation conceptuelle et mathématique., Cybergeo: European Journal of Geography, URL : http://cybergeo.revues.org/27150, 2015.

[PRO 15'] PROVITOLO D., REGHEZZA-ZITT M., Résilience et vulnérabilité : de l'opposition au continuum, REGHEZZA M., RUFAT S. (eds.), Editions ISTE, 2015.

[PRO 17] PROVITOLO D., SERRE D., BOSCHETTI L., Towards a process of resilience implementation : capacities, models, strategies, VINET F. (eds.), Ed. Iste, 2017.

[REG 15] REGHEZZA M., PROVITOLO D., LHOMME S., Définir la résilience : quand le concept résiste, in Résiliences, sociétés et territoires face à l'incertitude, aux risques et aux catastrophes, REGHEZZA-ZITT M., RUFAT S. (eds.), Editions ISTE, 2015.

[REG 15'] REGHEZZA M., RUFAT S., Resilience Imperative, Uncertainty, Risks and Disasters, REGHEZZA M., RUFAT S. (eds.), Editions ISTE, 2015.

[RES 10] RESILIENCE ALLIANCE, Assessing Resilience in Social-Ecological Systems: Workbook for Practitioners, Version 2.0, consultable à partir du site internet, http://www.resalliance.org, 2010.

[RIC 03] RICHEMOND A., La Résilience économique, Une chance de recommencement..., Edition d'Organisation, 231 p., 2003.

[ROB 10] ROBERT J., D'ERCOLE R., L'occupation et l'utilisation du sol en période de crise, Cybergeo : European Journal of Geography, URL : http://cybergeo.revues.org/26587, 2010.

[SEI 16] SEINE GRANDS LACS, Gestion de crue : nos ouvrages à la manœuvre, http://seinegrandslacs.fr/blog-hydrosolidaire/gestion-de-crue-nos-ouvrages-la-manoeuvre, 2016.

[SER 11] SERRE D., La ville résiliente aux inondations. Méthodes et outils d'évaluations, Mémoire d'Habilitations à Diriger les Recherches, Université Paris-Est, 2011.

[SER 12] SERRE D., LHOMME S., PEYRAS L., LAGANIER R., DIAB Y., Analyzing the civil engineering infrastructures to prioritize urban flood resilient actions, 7th International Conference on Water Sensitive Urban Design, 21-23 February 2012, Melbourne, Australia, 2012. 
[URD11] URGENCE REHABILITATION DEVELOPPEMENT (URD), Villes et crises., Sources documentaires de la conférence « La ville face aux crises » du 26 avril 2011, 2011.

[VIG 06] VIGNERON S., DÉGARDIN F., et al., Réduire la vulnérabilité des réseaux urbains aux inondations. Risques naturels majeurs. CERTU, C. d. l'Est and C. Sud-Ouest, Ministère de l'Ecologie et du Développement Durable: 112 p., 2006.

[WAL 04] WALKER B.H., HOLLING C.S., CARPENTER St R., KINZIG A., Resilience, adaptability and transformability in social-ecological systems, Ecology and Society, 9 p., 2004. 